\title{
Relationship between cardiovascular dysfunction and hyperglycemia in streptozotocin-induced diabetes in rats
}

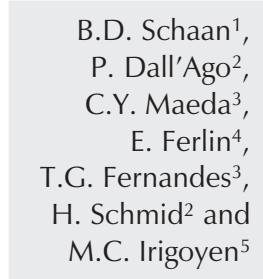

Correspondence

\section{B.D. Schaan}

Unidade de Pesquisa

Instituto de Cardiologia do

Rio Grande do Sul

Av. Princesa Isabel, 370

90620-001 Porto Alegre, RS

Brasil

Fax: +55-51-230-3600

E-mail: pesquisa@cardiologia.org.br

Publication supported by FAPESP.

Received August 13, 2003 Accepted August 3, 2004

\author{
'Instituto de Cardiologia do Rio Grande do Sul, \\ Fundação Universitária de Cardiologia, Porto Alegre, RS, Brasil \\ ${ }^{2}$ Fundação Faculdade Federal de Ciências Médicas de Porto Alegre, Porto Alegre, \\ RS, Brasil \\ ${ }^{3}$ Instituto de Biociências, Universidade Federal do Rio Grande do Sul, Porto Alegre, \\ RS, Brasil \\ ${ }^{4}$ Hospital de Clínicas de Porto Alegre, Porto Alegre, RS, Brasil \\ ${ }^{5}$ Laboratório de Hipertensão Experimental, Unidade de Hipertensão, Instituto do \\ Coração, Faculdade de Medicina, Universidade de São Paulo, São Paulo, SP, Brasil
}

\section{Abstract}

Streptozotocin (STZ)-induced diabetes in rats is characterized by cardiovascular dysfunction beginning 5 days after STZ injection, which may reflect functional or structural autonomic nervous system damage. We investigated cardiovascular and autonomic function, in rats weighing $166 \pm 4 \mathrm{~g}, 5-7,14,30,45$, and 90 days after STZ injection ( $\mathrm{N}=24,33,27,14$, and 13 , respectively). Arterial pressure (AP), mean AP (MAP) variability (standard deviation of the mean of MAP, SDMMAP), heart rate (HR), HR variability (standard deviation of the normal pulse intervals, SDNN), and root mean square of successive difference of pulse intervals (RMSSD) were measured. STZ induced increased glycemia in diabetic rats $v s$ control rats. Diabetes reduced resting HR from $363 \pm 12$ to $332 \pm 5$ bpm $(\mathrm{P}<0.05)$ 5 to 7 days after STZ and reduced MAP from $121 \pm 2$ to $104 \pm 5 \mathrm{mmHg}$ $(\mathrm{P}=0.007) 14$ days after STZ. HR and MAP variability were lower in diabetic vs control rats 30-45 days after STZ injection (RMSSD decreased from $5.6 \pm 0.9$ to $3.4 \pm 0.4 \mathrm{~ms}, \mathrm{P}=0.04$ and SDMMAP from $6.6 \pm 0.6$ to $4.2 \pm 0.6 \mathrm{mmHg}, \mathrm{P}=0.005)$. Glycemia was negatively correlated with resting AP and $\mathrm{HR}(\mathrm{r}=-0.41$ and $-0.40, \mathrm{P}<0.001)$ and with SDNN and SDMMAP indices $(\mathrm{r}=-0.34$ and $-0.49, \mathrm{P}<0.01)$. Even though STZ-diabetic rats presented bradycardia and hypotension early in the course of diabetes, their autonomic function was reduced only 30-45 days after STZ injection and these changes were negatively correlated with plasma glucose, suggesting a metabolic origin.

\section{Introduction}

Many clinical studies have demonstrated that chronic diabetic complications occur late after the onset of the disease, reflecting structural abnormalities in nerves, kidneys,
Key words

- Diabetes

- Streptozotocin

- Heart rate variability

- Rats

- Autonomic nervous system

- Arterial pressure variability

...... A. \footnotetext{
could represent a premature development of
}

retina, and cardiovascular system, strongly relating their appearance to the duration of diabetes and to glycemic control (1-3). However, some investigators have described the early appearance of abnormalities which 
these complications in autonomic and peripheral nerves $(4,5)$. These findings are consistent with the hypothesis that poor metabolic control is a major determinant of nervous damage. Indeed, other studies of type 1 diabetic patients have shown that autonomic nervous system abnormalities could be reversed when better metabolic control was obtained $(6,7)$.

The injection of streptozotocin (STZ) in rats leads to the development of a clinical syndrome characterized by hyperglycemia, excessive osmotic diuresis and loss of weight, which is similar to human diabetes. Moreover, the STZ-diabetic rat develops the usual chronic microvascular complications (nephropathy, peripheral and autonomic neuropathy) as observed in diabetic patients (810). Studies on 5-day STZ-diabetic rats from our laboratory have shown depressed vagal tone, reduction of vagal effect (11) and impaired tachycardic response to arterial pressure (AP) decreases (12). Fifteen days after STZ injection we observed impairment of the reflex bradycardia and tachycardia produced by vasopressor and vasodepressor agents, respectively $(13,14)$. Similar cardiovascular changes were described in this model (15) and there is evidence that some of these alterations are reversed by insulin therapy (16). These findings, associated with the impairment of baroreflex sensitivity, an excellent gauge of autonomic function, suggested the early development of autonomic dysfunction in these animals.

Since the autonomic nervous system modulates beat-to-beat fluctuations in heart rate (HR), methods to quantify HR and blood pressure variability have been evaluated as indicators of sympathetic and parasympathetic modulation of the cardiovascular system in humans (17) and in experimental models $(18,19)$. These methods seemed to detect early autonomic dysfunction at a time when other metabolic dysfunctional changes were not clearly observed. Indeed, the relationship between the abnormalities in auto- nomic modulation and glycemic control observed in humans has not been explored in diabetic animals.

The objective of the present study was to investigate cardiovascular and autonomic functions, evaluated by HR and blood pressure variability, and their relationship to glycemic control in STZ-induced diabetic rats at different times after STZ injection.

\section{Material and Methods}

In order to obtain a significant number of animals with diabetes of different duration, we grouped data from all cardiovascular experiments performed in STZ-diabetic rats in our laboratory from 2000 to 2001, assembling data from 111 male Wistar rats. The experiments were performed by researchers similarly trained in data collection, and control and diabetic rats were always evaluated simultaneously in order to minimize possible circadian and seasonal changes. The animals, weighing 150-280 g, were obtained from the Animal House of Universidade Federal do Rio Grande do Sul, Porto Alegre, RS, Brazil, and kept in small groups with free access to tap water and standard rat chow.

Animals were made diabetic (D) by iv injection of $50-60 \mathrm{mg} / \mathrm{kg} \mathrm{STZ}$ (Sigma, St. Louis, MO, USA) dissolved in sodium citrate buffer, $\mathrm{pH}$ 4.5. Controls (C) were injected with citrate buffer alone. Rats were fasted overnight before STZ administration. STZ-treated rats were used only when they developed elevated plasma glucose levels (confirmed by qualitative measurements of blood glucose $>300 \mathrm{mg} \% 48 \mathrm{~h}$ after injection). Metabolic control was evaluated on the basis of plasma glucose and animal weight at the end of the experiments. Rats were grouped according to the time of hemodynamic evaluation after STZ injection, with 24 (13C, 11D), 33 (17C, 16D), 27 (15C, 12D), 14 (7C, 7D), and 13 (8C, 5D) rats being evaluated after 5-7, 14, 30, 45, and 90 
days, respectively.

Catheters filled with saline were implanted under anesthesia into the femoral artery and vein (PE-10) for direct measurement of AP and drug administration, respectively. One day after catheter placement, the arterial cannula was connected to a straingauge transducer (P23Db; Gould-Statham, Oxnard, CA, USA) and blood pressure signals were recorded during a 40-min period with a microcomputer equipped with an analog-to-digital converter board (CODAS, 2$\mathrm{kHz}$ sampling frequency; Dataq Instruments, Inc., Akron, OH, USA). Rats were conscious and moved freely during the experiments. Recorded data were analyzed on a beat-tobeat basis. To evaluate mean AP (MAP) variability, we used the standard deviation of the mean of MAP (SDMMAP) (20), while HR variability was evaluated by calculating the following indices in the time domain: 1) root mean square of successive differences of pulse intervals (RMSSD), and 2) standard deviation of the normal pulse intervals (SDNN) (18). Each method employed to analyze HR variability reflects different patterns of variability: RMSSD reflects highfrequency short-term variations in $\mathrm{HR}$, or vagal activity; SDNN is the square root of the variance, which is mathematically equal to the total power of the signal. The total power corresponds to the contribution of all harmonic components (short-term and long- term variation) responsible for the variability.

Data are reported as means \pm SEM. Statistical significance was calculated by the Student $t$-test to compare unpaired data of D and $\mathrm{C}$ during each period after STZ injection. The relationship between metabolic control, measured by plasma glucose and the degree of autonomic modulation, measured by the indices cited above, was evaluated by calculating the Pearson correlation coefficient. Differences were considered to be significant at $\mathrm{P}<0.05$ for all tests.

\section{Results}

\section{Body weight and plasma glucose}

Body weights were similar in all experimental groups at baseline. Five to 7 days after STZ injection, the weight of diabetic rats was similar to that of controls, although tending to be lower. The weight of the diabetic animals was lower for all other diabetic groups compared to their controls after 14, 30, 45, and 90 days of diabetes. Plasma glucose levels measured after STZ injection were higher than after citrate buffer injection at all times studied (Table 1).

\section{Arterial pressure and heart rate}

Groups studied 5-7 and 14, 30, 45, and 90 days after the induction of diabetes pre-

Table 1. Characterization of diabetic (D) and control (C) rats 5-7, 14, 30, 45, and 90 days after STZ injection.

\begin{tabular}{|c|c|c|c|c|c|c|c|c|c|c|}
\hline & \multicolumn{2}{|c|}{ 5-7 days } & \multicolumn{2}{|c|}{14 days } & \multicolumn{2}{|c|}{30 days } & \multicolumn{2}{|c|}{45 days } & \multicolumn{2}{|c|}{90 days } \\
\hline & $D(11)$ & C (13) & $D(16)$ & C (17) & $D(12)$ & C (15) & $\mathrm{D}(7)$ & $C(7)$ & $\mathrm{D}(5)$ & C (8) \\
\hline Initial & $164 \pm 5$ & $167 \pm 6$ & $206 \pm 13$ & $199 \pm 14$ & $203 \pm 17$ & $157 \pm 4$ & $237 \pm 10$ & $251=$ & $225 \pm 11$ & $241 \pm 9$ \\
\hline Final weight (g) & $170 \pm 4$ & $195 \pm 9$ & $189 \pm 12^{*}$ & $235 \pm 15$ & $223 \pm 13^{*}$ & $264 \pm 5$ & $183 \pm 8^{*}$ & $297 \pm 11$ & $207 \pm 11^{*}$ & $301 \pm 10$ \\
\hline PG $(\mathrm{mg} / \mathrm{dl})$ & $332 \pm 6^{*}$ & $119 \pm 7$ & $466 \pm 42^{*}$ & $133 \pm 8$ & $486 \pm 40^{*}$ & $131 \pm 21$ & $409 \pm 47^{*}$ & $132 \pm 18$ & $258 \pm 48^{*}$ & $89 \pm 13$ \\
\hline $\mathrm{MAP}(\mathrm{mmHg})$ & $108 \pm 1$ & $112 \pm 2$ & $104 \pm 5^{*}$ & $121 \pm 2$ & $100 \pm 4^{*}$ & $114 \pm 3$ & $100 \pm 6^{*}$ & $115 \pm 3$ & $109 \pm 0.2$ & $114 \pm 2$ \\
\hline $\mathrm{HR}(\mathrm{bpm})$ & $332 \pm 5$ & $363 \pm 12$ & $334 \pm 16^{*}$ & $369 \pm 9$ & $317 \pm 12$ & $347 \pm 11$ & $285 \pm 7^{*}$ & $379 \pm 18$ & $323 \pm 30$ & $337 \pm 9$ \\
\hline
\end{tabular}

Data are reported as means \pm SEM for the number of animals indicated in parentheses. $\mathrm{PG}=$ plasma glucose; $\mathrm{MAP}=$ mean arterial pressure; $\mathrm{HR}$ $=$ heart rate.

${ }^{*} \mathrm{P}<0.05$ vs $\mathrm{C}$ rats (Student $t$-test). 
Figure 1. Standard deviation of the normal pulse intervals (SDNN, panel A), root mean square of successive difference of pulse intervals (RMSSD, panel B) and standard deviation of the mean of MAP (SDMMAP, panel $C$ ) in diabetic (D) and control (C) animals 5-7 (13C, 11D), 14 (17C, 16D), 30 (15C, 12D), 45 (7C, 7D), and 90 (8D, 5C) days after STZ injection. ${ }^{*} \mathrm{P}<0.05$ compared to the respective control (Student $t$-test).

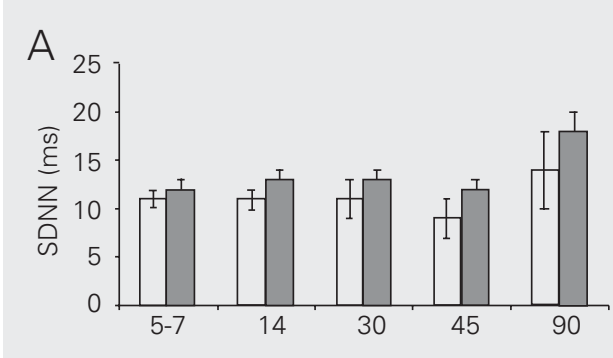

$B$
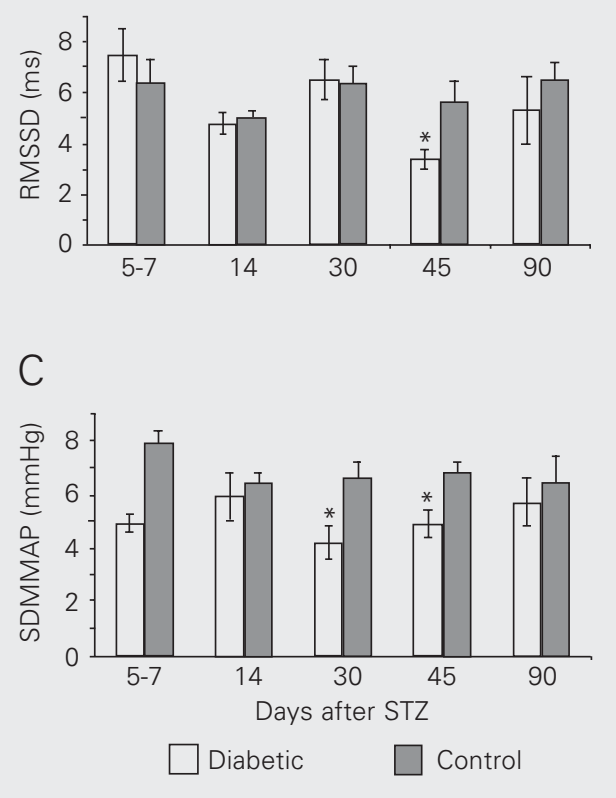

Figure 2. Scattergrams of mean arterial pressure (MAP, $\mathrm{N}=85$, panel $A)$, and heart rate $(H R, N=$ 87, panel B) versus plasma glucose in diabetic and control rats. Correlation was evaluated by the Pearson test.

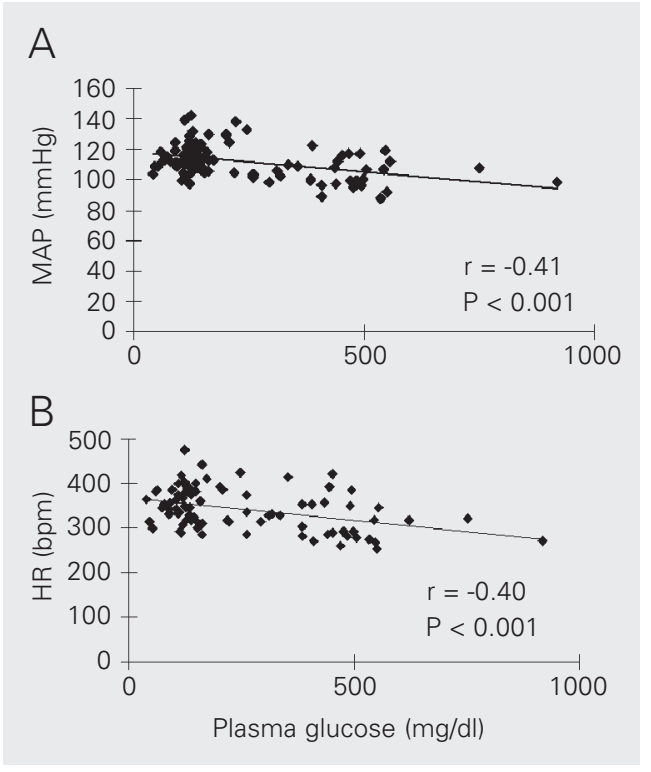

sented a significantly lower resting MAP compared to control at each time point of the study. HR was also lower in the D group compared to the $\mathrm{C}$ group at all times studied, although the difference was not statistically significant for all periods of diabetes evaluated (Table 1).

\section{Autonomic function}

The evaluation of autonomic function using indices of HR variability (SDNN and RMSSD) did not identify differences between the groups at 5-7, 14, 30, 45, and 90 days after STZ or citrate injection. The only exception was the RMSSD of the 45-day D animals, which was lower than their $\mathrm{C}$ group $(\mathrm{P}=0.04)$ (Figure 1). Also, MAP variability evaluated by the SDMMAP was significantly lower in the $\mathrm{D} v s \mathrm{C}$ groups at 30 and 45 days after induction $(4.2 \pm 0.6$ and $4.9 \pm 0.5$ vs $6.6 \pm 0.6$ and $6.8 \pm 0.4 \mathrm{mmHg}$ for $\mathrm{D}$ and $\mathrm{C}$, respectively; Table 1).

\section{Relationship between cardiovascular changes and plasma glucose}

By plotting the results obtained for all animals, D and C, it was possible to identify a negative relationship between plasma glucose levels and MAP $(r=-0.41, P<0.001)$, HR $(r=-0.40, P<0.001$; Figure $2 \mathrm{~A}$ and $\mathrm{B}$, respectively), SDNN $(r=-0.34, \mathrm{P}<0.001)$ and SDMMAP $(r=-0.49, \mathrm{P}<0.001$; Figure $3 \mathrm{~A}$ and $\mathrm{B}$, respectively). There was no relationship between RMSSD and plasma glucose levels $(r=-0.14, P=0.15)$.

\section{Discussion}

The present results demonstrate that STZdiabetic rats present changes in resting HR and AP very early in the course of diabetes. Nevertheless, autonomic function evaluated by time-domain indices of HR and MAP variability was reduced by diabetes only 30 to 45 days after STZ injection. Interestingly, 
all of these changes showed a negative correlation with plasma glucose.

Bradycardia in this animal model of diabetes has already been described by us and others $(11-13,18,21)$. Autonomic nervous system dysfunction, indicated by an increase in vagal tone or a decline in sympathetic tone to the heart may reduce HR, as suggested by Jackson and Carrier in 1983 (15), but previous data from our laboratory showed that 5 day diabetic rats had in fact reduced vagal function, whereas their sympathetic tone was not significantly affected (12). Perhaps the enhanced bradycardia induced by electrical vagal stimulation or methacholine administration could account in part for the final result of lower HR (22). This bradycardia may also be caused by a change in the electrophysiological properties of the sinoatrial node, since intrinsic HR is reduced (12). Previous results reported by our group (23) demonstrated that diabetes-induced bradycardia was attenuated by training, a change that was positively correlated with intrinsic HR. The metabolic improvement characteristic of exercise training may contribute to these changes.

Although some investigators have described a so-called STZ hypertension, this probably reflects discrepancies between the direct and indirect blood pressure measurements (24), since we and others, using the first method, consistently found hypotension in this animal model independently of the duration of diabetes $(11-13,18)$. Osmotic diuresis causing hypovolemia or myocardial dysfunction reducing contractile force are putative mechanisms of hypotension in STZ-diabetic rats $(23,25)$. Interestingly, both mechanisms reflect metabolic derangement. Moreover, an exaggerated pressor response to chronic hyperinsulinemia has been reported to occur in spontaneously hypertensive diabetic rats (26), an observation that allows us to expect the opposite change in blood pressure in response to hypoinsulinemia, which is characteristic of the STZ-diabetic rat.
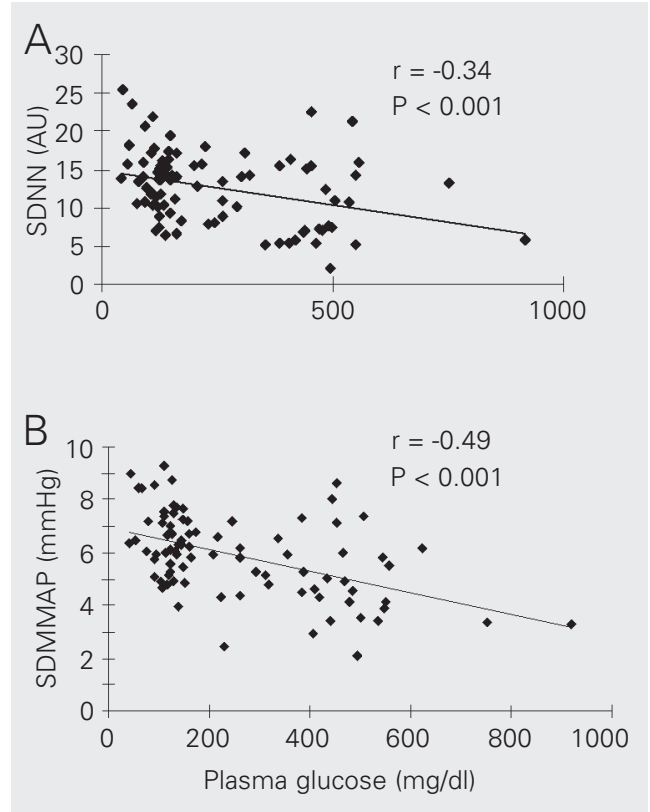

In humans, the earliest detectable feature of diabetic cardiac autonomic neuropathy is a defective parasympathetic control, represented by persistent resting tachycardia and loss of beat-to-beat variation during deep breathing (27), functional changes related to structural changes, as confirmed by postmortem studies (28). Fazan et al. (19) suggested that impairment of cardiac parasympathetic nerve function is present early in the course of diabetes in this animal model, as indicated by decreased HR variability. However, other investigators doubt its occurrence before diabetes has been present for some time (29). Indeed, previous results from our group, evaluating MAP variability by the three-dimensional return map, showed that rats with short-term STZ diabetes even present a normal autonomic control of HR and MAP (18). The present results did not demonstrate any change in SDNN, the same index used by Fazan et al. (19) to assess parasympathetic autonomic neuropathy from 5 to 90 days of diabetes duration. Another study, although evaluating spontaneously hypertensive diabetic rats, analyzed the alterations in AP and HR variability using spectral analysis approaches and found a
Figure 3. Scattergrams of the standard deviations of the normal pulse intervals (SDNN, $N=$ 85, panel A) and of the mean of MAP (SDMMAP, $N=86$, panel $B)$ versus plasma glucose in diabetic and control rats. $A U=$ arbitrary units. Correlation was evaluated by the Pearson test. 
reduced variability of AP without changes in HR variability in diabetic animals (30). This disagreement may be accounted for by the differences in the sensitivity of the methods used to evaluate autonomic dysfunction, i.e., time domain or frequency domain indices. The differences between the current results and previous ones obtained by our group could also be accounted for by different methodologies. Disturbances in autonomic function were observed in 5-day diabetic rats when evaluated by baroreflex sensitivity and after autonomic blockade with propranolol and atropine $(11,12)$, which could be more sensitive than the indices used in the present study.

Spontaneous fluctuations in MAP and HR have been used to detect autonomic dysfunction in humans (17). The reduced MAP variability, evaluated by SDMMAP, observed in the diabetic groups in the present study may indicate early functional autonomic cardiovascular dysfunction (31). The indices used to evaluate MAP and HR variability were negatively correlated with plasma glucose, suggesting that MAP and HR could be altered by diabetic metabolic decompensation. Accordingly, in spontaneously hypertensive diabetic rats, reflex bradycardic index and the power of low frequency oscillations of systolic arterial pressure are inversely related to blood glucose, reinforcing the crucial role of metabolic changes in the cardiovascular disorder in this model (30). Hicks et al. (21), in 1998, observed the same tendencies as observed here: a decline in the magnitude of the circadian variation and decreased sympathetic and parasympathetic nervous tone to the heart after STZ injection, changes which were reversed by insulin treatment.

It is unlikely that bradycardia and hypotension could be the result of autonomic neural structural injury; instead, they could represent autonomic neural dysfunction caused by metabolic changes induced by STZ diabetes. This is supported by the nega- tive correlation observed between MAP and HR with plasma glucose. Indeed, other investigators have demonstrated that bradycardia and hypotension induced by experimental diabetes can be prevented by insulin treatment $(16,30)$. Our group observed reduced intrinsic HR in short-term STZ diabetes, which could be the result of metabolic injury to pacemaker cells (11). Reduced motor nerve conduction velocity is also normalized by insulin treatment in this animal model (32). These data suggest that these early phenomena constitute a stage of neurological dysfunction distinct from "true" neuropathy (28). In fact, other investigators did not find structural abnormalities in the vagus nerve, which consists of parasympathetic preganglionic fibers, or enteric ganglia, which consist of parasympathetic neurons in STZdiabetic rats with a 6- to 12-month duration of diabetes $(33,34)$. Conversely, relevant structural changes characterized by axonal dystrophy did occur in the sympathetic preganglionic nerve fibers, but these changes were observed 12 months after STZ injection (34).

Several mechanisms have been considered to be involved in the pathogenesis of diabetic neuropathy in the STZ-diabetic rat, but hyperglycemia is always implicated. Structural changes occurring in peripheral nerves resemble those of human diabetic neuropathy and are preceded by hyperglycemia-induced biochemical abnormalities. Recent evidence has emphasized the importance of vascular dysfunction, driven by metabolic insults to the nerves, as a cause of diabetic neuropathy. Non-enzymatic glycosylation of myelin components, reduction of endoneural blood flow, increased oxygen free radical activity, or production and deprivation of the nerve growth factor are also involved $(32,35)$.

In agreement with our results, Burger et al. (36) described the reversibility of early cardiac autonomic neuropathy assessed by power spectral analysis of HR variability by 
glycemic control in type 1 diabetic patients, and comparable results were also published by Muhr-Becker et al. (37) in a study evaluating cardiac sympathetic dysinnervation scintigraphically in a similar group of patients.

A direct effect of hyperglycemia on vascular and myocardial cells should also be considered as a cause for cardiovascular dysfunction in this model. The protein kinase $\mathrm{C}$ (PKC) pathway, which is activated by hyperglycemia, has been recently recognized as an important mechanism in the development of diabetic complications including cardiomyopathy and angiopathy (38). Up-regulation of PKCß2 was demonstrated in the heart and aorta at both the transcriptional and translational levels during the early stages of experimental diabetes, suggesting its role in the diabetic injury to the cardiovascular system (39).

Our results demonstrate some well-known cardiovascular alterations presented by a commonly used diabetic model, the STZdiabetic rat, such as hypotension and bradycardia, and transitory autonomic index derangements. Considering data reported by other investigators who demonstrated that autonomic nerve structural lesions do not appear as early as these functional changes, we question whether these changes could be assigned to early development of autonomic neuropathy in this model, but we accept the presence of reversible neurological dysfunction. The negative correlation observed between cardiovascular dysfunction and plasma glucose is in accordance with these observations.

\section{References}

1. Pirart J (1984). Glycaemic control and prevention of complications. Minerva Endocrinology, 9: 55-58.

2. The Diabetes Control and Complications Trial Research Group (1993). The effect of intensive treatment of diabetes on the development and progression of long term complications in insulin-dependent diabetes mellitus. New England Journal of Medicine, 329: 977-986.

3. UK Prospective Diabetes Study (UKPDS) Group (1998). Intensive blood-glucose control with sulphonylureas or insulin compared with conventional treatment and risk of complications in patients with type 2 diabetes (UKPDS 33). Lancet, 352: 837-853.

4. Pfeifer MA, Weinberg CR, Cook DL, Reenan A, Halter JB, Ensinck JW \& Porte Jr D (1984). Autonomic neural dysfunction in recently diagnosed diabetic subjects. Diabetes Care, 7: 447-453.

5. Young RJ, Ewing DJ \& Clarke BF (1983). Nerve function and metabolic control in teenage diabetics. Diabetes, 32: 142-147.

6. Hreidarsson AB (1981). Acute, reversible autonomic nervous system abnormalities in juvenile insulin-dependent diabetes: A pupillographic study. Diabetologia, 20: 475-481.

7. Ferreira SR, Cesarini PR, Vivolo MA \& Zanella MT (1998). Abnormal nocturnal blood pressure fall in normotensive adolescents with insulin-dependent diabetes is ameliorated following glycemic improvement. Brazilian Journal of Medical and Biological Research, 31: 523-528.

8. Jensen PK, Sandahl Christiansen J, Steven K \& Parving H-H (1981). Renal function in streptozotocin-diabetic rats. Diabetologia, 21: 409414.

9. Cameron NE, Cotter MA \& Maxfield EK (1993). Anti-oxidant treatment prevents the development of peripheral nerve dysfunction in streptozotocin-diabetic rats. Diabetologia, 36: 299-304.
10. Schmidt RE \& Plurad SB (1986). Ultrastructural and biochemical characterization of autonomic neuropathy in rats with chronic streptozotocin diabetes. Journal of Neuropathology and Experimental Neurology, 45: 525-544.

11. Maeda CY, Fernandes TG, Timm HB \& Irigoyen MC (1995). Autonomic dysfunction in short-term experimental diabetes. Hypertension, 26: 1000-1004.

12. Maeda CY, Fernandes TG, Lulhier F \& Irigoyen MC (1995). Streptozotocin diabetes modifies arterial pressure and baroreflex sensitivity in rats. Brazilian Journal of Medical and Biological Research, 28: 497-501.

13. Dall'Ago P, Fernandes TG, Machado UF, Belló AA \& Irigoyen MC (1997). Baroreflex and chemoreflex dysfunction in streptozotocindiabetic rats. Brazilian Journal of Medical and Biological Research, 30: 119-124.

14. De Angelis K, Schaan BD, Maeda CY, Dall'Ago P, Wichi RB \& Irigoyen MC (2002). Cardiovascular control in experimental diabetes. Brazilian Journal of Medical and Biological Research, 35: 10911100

15. Jackson CV \& Carrier GO (1983). Influence of short-term experimental diabetes on blood pressure and heart rate in response to norepinephrine and angiotensin II in the conscious rat. Journal of Cardiovascular Pharmacology, 5: 260-265.

16. Chang KSK \& Lund DD (1986). Alterations in the baroreceptor reflex control of heart rate in streptozotocin diabetic rats. Journal of Molecular and Cellular Cardiology, 18: 617-624.

17. Task Force of the European Society of Cardiology and the North American Society of Pacing and Electrophysiology (1996). Heart rate variability: standards of measurement, physiological interpretation, and clinical use. Circulation, 93: 1043-1065. 
18. Schaan BD, Maeda CY, Timm H, Medeiros S, Moraes R, Ferlin E, Fernandes TG, Ribeiro JP, Schmid H \& Irigoyen MC (1997). Time course of changes in heart rate and blood pressure variability in streptozotocin-induced diabetic rats treated with insulin. Brazilian Journal of Medical and Biological Research, 30: 1081-1086.

19. Fazan R, Ballejo G, Salgado MC, Moraes MFD \& Salgado HC (1997). Heart rate variability and baroreceptor function in chronic diabetic rats. Hypertension, 30: 632-635.

20. Mancia G \& Zanchetti A (1986). Blood pressure variability. In: Zanchetti A \& Tarazi RC (Editors), Handbook of Hypertension. Vol. 7. Pathophysiology of Hypertension. Cardiovascular Aspects. Elsevier Science Publishers B.V., Amsterdam, The Netherlands, 125-152.

21. Hicks KK, Seifen E, Stimers JR \& Kennedy RH (1998). Effects of streptozotocin-induced diabetes on heart rate, blood pressure and cardiac autonomic nervous control. Journal of the Autonomic Nervous System, 69: 21-30.

22. Dall'Ago $\mathrm{P}$, Silva $\mathrm{VO}$, De Angelis $\mathrm{KL}$, Irigoyen $\mathrm{MC}$, Fazan Jr R \& Salgado HC (2002). Reflex control of arterial pressure and heart rate in short-term streptozotocin diabetic rats. Brazilian Journal of Medical and Biological Research, 35: 843-849.

23. De Angelis KLD, Oliveira AR, Dall'Ago P, Peixoto LRA, Gadonsky G, Lacchini S, Fernandes TG \& Irigoyen MC (2000). Effects of exercise training on autonomic and myocardial dysfunction in streptozotocindiabetic rats. Brazilian Journal of Medical and Biological Research, 33: 635-641

24. Kusaka M, Kishi K \& Sokabe H (1987). Does so-called streptozotocin (STZ) hypertension exist in rats? Hypertension, 10: 517-521.

25. Hebden RA, Gardiner SM, Bennett T \& MacDonald IA (1986). The influence of streptozotocin-induced diabetes mellitus on fluid and electrolyte handling in rats. Clinical Science, 70: 111-117.

26. Brands MW, Garrity CA, Holman MG \& Hall JE (1994). Exaggerated pressor and chronotropic response to chronic hyperinsulinemia in SH versus WKY rats. American Journal of Hypertension, 7: 75-81.

27. Ewing DJ, Campbell W \& Clarke BF (1980). The natural history of diabetic autonomic neuropathy. Quarterly Journal of Medicine, 193: 95-108.

28. Duchen LW, Anjorin A, Watkins P \& Mackay JD (1980). Pathology of autonomic neuropathy in diabetes mellitus. Annals of Internal Medicine, 92: 301-303.

29. Hounsom L \& Tomlinson DR (1997). Does neuropathy develop in animal models? Clinical Neuroscience, 4: 380-389.

30. Farah VMA, De Angelis K, Fiorino $\mathrm{P}$, Joaquim LF, Ayala E, Fazan Jr R, Morris M \& Irigoyen MC (2003). Autonomic modulation of arterial pressure and heart rate variability in hypertensive diabetic rats. Annals of the XV Scientific Meeting of the Inter-American Society of Hypertension, San Antonio, TX, USA, April 27-30, 92 (P127).

31. Mésangeau D, Laude D \& Elghozi J-L (2000). Early detection of cardiovascular autonomic neuropathy in diabetic pigs using blood pressure and heart rate variability. Cardiovascular Research, 45: 889-899.

32. Greene DA, De Jesus PVJ \& Winegrad Al (1975). Effects of insulin and dietary myoinositol on impaired peripheral motor nerve conduction velocity in acute streptozotocin diabetes. Journal of Clinical Investigation, 55: 1326-1336.

33. Schmidt RE, Plurad SB \& Modert CW (1983). Experimental diabetic autonomic neuropathy characterization in streptozotocin-diabetic Sprague-Dawley rats. Laboratory Investigation, 49: 538-552.

34. Kniel PC, Junker U, Perrin IV, Bestetti GE \& Rossi GL (1986). Varied effects of experimental diabetes on the autonomic system of the rat. Laboratory Investigation, 54: 523-530.

35. Lee PG, Hohman TC, Cai F, Regalia J \& Helke CJ (2001). Streptozotocin-induced diabetes causes metabolic changes and alterations in neurotrophin content and retrograde transport in the cervical vagus nerve. Experimental Neurology, 170: 149-161.

36. Burger AJ, Weinrauch LA, D'Elia JA \& Aronson D (1999). Effect of glycemic control on heart rate variability in type I diabetic patients with cardiac autonomic neuropathy. American Journal of Cardiology, 84: 687-691.

37. Muhr-Becker D, Weiss M, Tatsch K, Wolfram G, Standl E \& Schnell O (1999). Scintigraphically assessed cardiac sympathetic dysinnervation in poorly controlled type 1 diabetes mellitus: one-year followup with improved metabolic control. Experimental and Clinical Endocrinology and Diabetes, 107: 306-312.

38. Idris I, Gray S \& Donnelly R (2001). Protein kinase C activation: isozyme-specific effects on metabolism and cardiovascular complications in diabetes. Diabetologia, 44: 659-673.

39. Guo M, Wu MH, Korompai F \& Yuan SY (2003). Upregulation of PKC genes and isozymes in cardiovascular tissues during early stages of experimental diabetes. Physiological Genomics, 12: 139-146. 